\title{
Comparando a Psicoterapia Psicodinâmica de uma menina com modelos de Psicoterapia Psicodinâmica e Terapia Cognitivo- Comportamental
}

\author{
Guilherme Pacheco Fiorini ${ }^{1,2,3}$ \\ Marina Bento Gastaud ${ }^{4}$ \\ Vera Regina Röhnelt Ramires \\ ${ }^{1}$ Universidade do Vale do Rio dos Sinos, $R S$, Brasil \\ ${ }^{2}$ University College London, Londres, Reino Unido \\ ${ }^{3}$ Anna Freud National Centre for Children and Families, Londres, Reino Unido \\ https://orcid.org/0000-0003-3107-4611 \\ ${ }^{4}$ Contemporâneo - Instituto de Psicanálise e Transdisciplinaridade, $R S$, Brasil \\ https://orcid.org/0000-0002-4040-0995 \\ ${ }^{5}$ Universidade do Vale do Rio dos Sinos, RS, Brasil \\ https://orcid.org/0000-0002-1760-7154
}

\begin{abstract}
Resumo
Esse estudo teve como objetivo comparar o processo da Psicoterapia Psicodinâmica (PP) de uma criança com sintomas internalizantes aos protótipos brasileiros de terapia cognitivo-comportamental (TCC) e de PP para crianças com transtornos internalizantes e externalizantes. Para isso, pares de juízes avaliaram todas as sessões $(\mathrm{n}=40)$ da psicoterapia de uma menina com sintomas internalizantes utilizando o Child Psychotherapy Q-Set (CPQ). Os graus de similaridade das sessões aos protótipos brasileiros de psicoterapia foram calculados via correlação de Pearson. Como resultado, o tratamento apresentou similaridade significativa simultaneamente aos protótipos da TCC e da PP para crianças com transtornos internalizantes. Não foi encontrada similaridade significativa ao protótipo da PP para crianças com transtornos externalizantes. Verificou-se que os protótipos brasileiros de psicoterapia de crianças mostraram-se uma ferramenta útil na avaliação desse processo terapêutico. Os dados apresentados indicam que, na prática, as características das psicoterapias de crianças se sobrepõem e os processos apresentam um caráter integrativo.
\end{abstract}

Palavras-chave: processos psicoterapêuticos, psicoterapia da criança, psicoterapia psicanalítica, terapia cognitivo-comportamental.

\section{Comparing the Psychodynamic Psychotherapy of a girl with models of Psychodynamic Psychotherapy and Cognitive-Behavioral Therapy}

\begin{abstract}
This study aimed to compare the psychodynamic psychotherapy (PDT) process of a child with internalising symptoms to Brazilian prototypes of Cognitive-behavioural therapy (CBT) and PDT for children with internalizing and externalizing disorders. For that purpose, pairs of judges rated all PDT sessions $(n=40)$ of a girl with internalising symptoms using the Child Psychotherapy $Q-S e t(C P Q)$. The degrees of similarity to the Brazilian prototypes were assessed via Pearson correlation. The results indicated that the treatment presented simultaneous significant similarity to the prototypes of CBT and PDT for children with internalising disorders. The simila-
\end{abstract}


rity to the prototype of PDT for children with externalising disorders was not significant in this treatment. The findings suggest that the Brazilian prototypes of child psychotherapy were a useful assessment tool for this psychotherapeutic process. The data presented indicate that, on clinical practice, psychotherapies features overlap, and child psychotherapy processes have an integrative nature.

Keywords: psychotherapeutic processes, child psychotherapy, psychoanalytic psychotherapy, cognitive behavioral therapy.

\section{Comparando la Psicoterapia Psicodinámica de una niña con modelos de Psicoterapia Psicodinámica y Terapia Cognitivo-Conductual}

\section{Resumen}

Este estudio tuvo por objetivo comparar el proceso de la psicoterapia psicodinámica (PP) de una niña con síntomas internalizantes con prototipos brasileños de terapia cognitiva-conductual (TCC) y PP para niños con trastornos internalizantes y externalizantes. Para eso, pares de jueces calificaron todas las sesiones de la PP $(n=40)$ de una niña con síntomas internalizantes utilizando el Child Psychotherapy Q-Set (CPQ). Los grados de similitud con los prototipos brasileños se evaluaron mediante la correlación de Pearson. Como resultado, el tratamiento presentó una similitud significativa simultánea con los prototipos de TCC y PP para niños con trastornos internalizantes. La similitud con el prototipo de la PP para niños con trastornos externalizantes no fue significativa en este tratamiento. Los prototipos brasileños de psicoterapia infantil demostraron ser una herramienta útil para la evaluación de este proceso psicoterápico. Los datos presentados indican que, en la práctica clínica, las psicoterapias se superponen, y los procesos de psicoterapia infantil tienen una naturaleza integradora.

Palabras-chave: procesos psicoterapéuticos, psicoterapia infantil, psicoterapia psicoanalítica, terapia cognitiva-conductista.

Os transtornos internalizantes estão entre os mais prevalentes em crianças no mundo (Polanczyk, Salum, Sugaya, Caye, \& Rohde, 2015). Nesses casos, as problemáticas vivenciadas envolvem primordialmente o humor e as emoções (Kovacs \& Devlin, 1998) e, dentre os principais sintomas identificados, encontram-se retraimento, queixas somáticas e síndromes de ansiedade e depressão. Devido a essas crianças sentirem os seus problemas "dentro de si" e não afetarem diretamente o ambiente (Wilkinson, 2009), a identificação desses quadros tende a ser mais sutil (Recktenvald, Mallmann, Schmidt, Fiorini, \& Cappellari, 2016). Contudo, o não tratamento de condições internalizantes pode acarretar a pobre interação com pares, além de prejuízos nas esferas educacional e social (Oland \& Shaw, 2005; Organização Mundial da Saúde [OMS], n.d.), configurando uma demanda urgente de tratamentos para essa população.

Nesse sentido, revisões recentes da literatura vêm indicando que a psicoterapia psicodinâmica (PP) e a terapia cognitivo-comportamental (TCC) são efetivas para o tratamento de crianças, principalmente as que apresentam quadros internalizantes (Chorpita et al., 2011; Midgley, O’Keeffe, French, \& Kennedy, 2017; Palmer, Nascimento,
\& Fonagy, 2013). No entanto, apesar dos resultados dessas psicoterapias serem promissores, ainda pouco se sabe sobre "como" e "por que" as mudanças terapêuticas ocorrem (Kazdin, 2009; Palmer et al., 2013). Frente a essa demanda, pesquisas referentes ao processo terapêutico buscam responder a essas perguntas. Elas caracterizam-se pela mensuração de características presentes ao longo da psicoterapia que podem contribuir para alcançar os resultados desejados ou dificultá-los (Kazdin, 2009).

Considerando a multiplicidade de fatores envolvidos no processo terapêutico, há o debate sobre os fatores comuns e específicos das psicoterapias como agentes de mudança (Ablon \& Jones, 1998; Leonardi \& Meyer, 2015). Por um lado, alguns autores defendem que os resultados obtidos por meio das intervenções terapêuticas dão-se principalmente por fatores que deveriam estar presentes em todas as psicoterapias, como a capacidade empática do terapeuta, a sua postura de aceitação e a aliança terapêutica (Wampold, 2015). Por outro lado, há autores que destacam a primazia de fatores específicos de determinadas abordagens, constituídos por atitudes deliberadas do terapeuta que são baseadas em fundamentos teóricos, incluindo intervenções e técnicas (Jones, 
Cumming, \& Horowitz, 1988; Shedler, 2010). Nesse contexto, os estudos de protótipos em psicoterapia são ferramentas que contribuem na investigação de fatores comuns e específicos presentes no processo terapêutico.

A metodologia de protótipos foi cunhada por Ablon e Jones (1998) e consiste no desenvolvimento de modelos ideais ou típicos dos processos psicoterápicos a partir da opinião de experts, terapeutas com ampla experiência clínica e que são referência em suas respectivas abordagens psicoterápicas. Para a construção dos protótipos, experts selecionados preenchem o Psychotherapy Process Q-Set (PQS; Jones, 1985), um instrumento formado por 100 itens que descrevem o que podem acontecer em uma sessão terapêutica com pacientes adultos, buscando descrever tais sessões ideais ou típicas. O emprego do PQS para a formulação de protótipos auxiliou na criação de modelos empiricamente aplicáveis, úteis para se identificar características do processo terapêutico de diferentes abordagens; para verificar se os tratamentos seguem, na prática, o que propõem; e investigar se os resultados obtidos estão associados ao grau de similaridade entre o processos e os protótipos (Ablon \& Jones, 1998; Leichsenring et al., 2016).

A partir de estudos que adotaram protótipos para a investigação de processos terapêuticos reais com pacientes adultos, evidenciou-se que as psicoterapias, na prática, nem sempre correspondem aos seus pressupostos teóricos, e que um mesmo tratamento pode incluir aspectos prototípicos de diferentes abordagens (Ablon \& Jones, 2002; Serralta $\&$ Ablon, 2016). Além disso, achados indicaram que os mecanismos que explicam o sucesso de um determinado tratamento podem ser melhor explicados por fatores prototípicos de outros tratamentos (Ablon \& Jones, 1998; Serralta, Pole, Nunes, Eizirik, \& Olsen, 2010).

Expandindo essa metodologia para o estudo do processo terapêutico com crianças, Goodman, Midgley e Schneider (2016) convidaram experts de diferentes nacionalidades para descrever sessões ideais de psicoterapia com pacientes crianças. Utilizando o Child Psychotherapy Q-Set (CPQ; Ramires \& Schneider,
2016), adaptação do PQS para a avaliação do processo psicoterapêutico com crianças, foram investigadas as concepções prototípicas da PP, TCC e da abordagem baseada na mentalização (FR) de crianças. A FR é uma forma de tratamento que focaliza o desenvolvimento da capacidade de mentalização, construto que descreve a capacidade individual de compreender os comportamentos próprios e alheios em relação a estados mentais subjacentes (Fonagy, Bateman, \& Luyten, 2012).

A partir das avaliações dos experts, Goodman et al. (2016) formularam três protótipos de psicoterapias de crianças, um para cada abordagem apreciada no estudo. Ademais, a partir das análises estatísticas empregadas, os autores identificaram que os processos prototípicos das psicoterapias de crianças são conceptualmente mais similares entre si do que os de adultos, sendo o desenvolvimento da capacidade de mentalização um possível fator comum entre as diferentes abordagens (Goodman et al., 2016).

Assim como nos estudos com pacientes adultos, investigações que analisaram psicoterapias reais a partir dos protótipos desenvolvidos por Goodman et al. (2016) indicaram que as psicoterapias de crianças não seguem, necessariamente, as suas diretrizes teóricas, e que esses tratamentos tendem a apresentar um caráter integrativo (Gastaud, Carvalho, Goodman, \& Ramires, 2015; Goodman et al., 2016; Goodman, Reed, \& Athey-Lloyd, 2015; Ramires, Godinho, \& Goodman, 2017). Da mesma forma, essas pesquisas vêm indicando que o grau de similaridade dos protótipos é impactado pelas características da díade, e que o desenvolvimento da capacidade de mentalização - identificado pela semelhança ao protótipo da FR - está presente em diferentes tratamentos (Gastaud et al., 2015; Goodman et al., 2015; Ramires et al., 2017).

Buscando aprofundar as investigações de protótipos de psicoterapias com crianças e reduzir limitações transculturais, Fiorini e Ramires (2019) desenvolveram protótipos brasileiros de TCC e PP específicos para crianças com transtornos internalizantes e externalizantes. Nessa pesquisa, nove experts de TCC e nove experts de PP responderam o CPQ a fim de descrever o processo 
prototípico de sua respectiva abordagem para crianças com transtornos internalizantes e, após, preencheram os mesmos itens buscando descrever o processo prototípico com crianças com transtornos externalizantes.

Nesse estudo, os autores identificaram três protótipos evidenciados por meio de análise fatorial- que explicavam $66,41 \%$ da variação dos itens do CPQ. Um deles concentrou todas as avaliações dos experts em TCC, enquanto os protocolos dos experts em PP para crianças com transtornos internalizantes e de PP para crianças com transtornos externalizantes formaram dois fatores distintos.

O protótipo brasileiro da TCC de crianças caracterizou um tratamento em que o terapeuta ativamente estrutura a sessão e se comporta de maneira didática, buscando auxiliar a criança a manejar os seus sentimentos e modificar distorções em suas crenças. Considerando as análises realizadas, não houve distinção empiricamente identificável entre o processo prototípico da TCC para crianças com transtornos internalizantes e externalizantes (Fiorini \& Ramires, 2019).

Por outro lado, as avaliações em PP caracterizaram dois protótipos estatisticamente diferenciáveis. O protótipo da PP para crianças com transtornos internalizantes configurou um tratamento conduzido por um terapeuta neutro, que interpreta os conteúdos inconscientes e o brincar da criança, além de assinalar suas defesas. Já o protótipo da PP para crianças com transtornos externalizantes descreveu um processo cuja ênfase é o trabalho na relação terapêutica e suas possíveis conexões com outros relacionamentos fora do setting. Ademais, foi destacado nesse protótipo que o terapeuta deve manter uma atitude tolerante frente aos fortes afetos e impulsos da criança (Fiorini \& Ramires, 2019).

Apesar desses achados contribuírem para o entendimento dos processos psicoterapêuticos da TCC e da PP para crianças com diferentes sintomatologias a partir da opinião de experts brasileiros, os protótipos desenvolvidos ainda não foram utilizados para avaliar tratamentos reais. Além disso, destaca-se que nenhuma publicação empregou a metodologia dos protótipos para investigar toda a extensão do processo terapêutico de uma criança com um quadro internalizante. Nesse sentido, o emprego dos protótipos brasileiros na investigação de uma psicoterapia real seria relevante para verificar se esses modelos são capazes de captar aspectos-chave dos processos terapêuticos, assim contribuindo para o estudo das psicoterapias infantis.

Tendo isso em vista, o presente estudo teve como objetivo investigar o processo da psicoterapia psicodinâmica de uma criança com sintomas internalizantes em relação à sua similaridade aos protótipos brasileiros da PP e da TCC. Foram testadas as seguintes hipóteses: (1) o tratamento apresentaria similaridade significativa ao protótipo da PP para crianças com transtornos internalizantes, considerando a abordagem teórica da psicoterapia proposta e a sintomatologia da paciente; (2) o tratamento não apresentaria similaridade significativa ao protótipo da TCC; e (3) o tratamento não apresentaria similaridade significativa ao protótipo da PP para crianças com transtornos externalizantes.

\section{Método}

\section{Delineamento}

Esse estudo faz parte de uma pesquisa maior, que visa investigar o processo psicoterapêutico psicodinâmico de crianças em relação aos seus resultados e a protótipos de psicoterapia. O presente recorte foi constituído por um delineamento longitudinal e correlacional (Sampieri, Collado, \& Lucio, 2014), baseado no método de Estudos de Caso Sistemático (Edwards, 2007). Todas as diretrizes éticas foram respeitadas, sendo este projeto aprovado pelo Comitê de Ética em Pesquisa da Universidade do Vale do Rio dos Sinos (Resolução 039/2012).

\section{Participantes}

Os participantes desse estudo foram uma menina e sua terapeuta. A paciente (pseudônimo Alice) tinha oito anos de idade no início da psicoterapia, cursava o terceiro ano do ensino fundamental e morava com 
sua mãe e irmã, dois anos mais nova. Alice recebeu indicação para psicoterapia pelo seu neuropediatra, por apresentar dores físicas sem causa identificável por exames médicos. Seus sintomas surgiram cinco meses após a morte de seu pai, que ocorreu devido a uma complicação de saúde súbita e inesperada. A morte do pai de Alice acarretou dificuldades financeiras, e a paciente teve de mudar de escola dois meses antes do início do tratamento. Em relação à troca de escola, Alice relatava sentir saudades dos colegas antigos, dificuldade em fazer novas amizades e descontentamento com uma suposta falta de organização na escola nova. Ela chorava frequentemente e relutava em ir às aulas. A partir do relato da mãe e de sua professora, Alice era uma menina carinhosa, rígida e perfeccionista. Segundo a mãe da paciente, a menina também tinha dificuldade em lidar com assuntos sexuais: sentia-se desagradada ao ver demonstrações públicas de afeto e ao ouvir sobre relações amorosas entre adultos. A partir da avaliação inicial, mensurada pelo Child Behaviour Checklist (CBCL) (Achenbach, 1991) preenchido pela mãe, a paciente apresentava escore clínico para problemas internalizantes (Tabela 1).

A psicoterapeuta de Alice tinha 10 anos de experiência clínica, era especialista, mestre, doutora e realizava pós-doutorado na área de psicoterapia psicodinâmica da infância. Ela supervisionava o caso da menina e realizava análise pessoal. Durante o período da psicoterapia, a terapeuta de Alice engravidou.

\section{Tabela 1:}

\section{CBCL 6-18/Escala de síndromes}

\begin{tabular}{|l|c|l|}
\hline & Pontuação & Classificação \\
\hline Internalizantes & & \\
\hline Ansiedade/depressão & 14 & Clínico \\
\hline Retraimento/depressão & 7 & Clínico \\
\hline Queixas somáticas & 8 & Clínico \\
\hline Externalizantes & & \\
\hline
\end{tabular}

\begin{tabular}{|l|c|c|}
\hline Comportamento & 2 & Não clínico \\
\hline $\begin{array}{l}\text { Comportamento } \\
\text { agressivo }\end{array}$ & 6 & Não clínico \\
\hline Neutro & 7 & Não clínico \\
\hline Problemas sociais & 7 & Não clínico \\
\hline $\begin{array}{l}\text { Problemas de } \\
\text { pensamento }\end{array}$ & 3 & Não clínico \\
\hline Problemas de atenção & & \\
\hline
\end{tabular}

Classificação avaliada a partir dos parâmetros ASEBA para meninas entre 6 a 11 anos.

\section{Tratamento}

O tratamento foi constituído por 40 sessões individuais com a criança, oito sessões com a sua mãe e uma sessão em conjunto com a mãe e a paciente. Os encontros ocorreram em um intervalo de 14 meses, e a frequência da psicoterapia foi predominantemente semanal. Entre as sessões 33 e 34 houve uma interrupção de três meses, constituindo o período de licença maternidade da terapeuta. No retorno da licença maternidade, foi realizada uma sessão com a presença da paciente e sua mãe. Nesse encontro, foi contratado o final da psicoterapia, e sete sessões para trabalhar a despedida entre a díade foram combinadas. A mãe da paciente relatou que os problemas que levaram Alice a tratamento já haviam sido resolvidos, e que situações financeiras, familiares e logísticas impossibilitavam a continuidade da psicoterapia. A paciente manifestou interesse em permanecer no tratamento, e a terapeuta identificou demandas que ainda precisavam ser trabalhadas, mas ambas reconheceram os ganhos terapêuticos alcançados até então. As sessões de psicoterapia ocorreram no consultório particular da terapeuta na região Sul do Brasil e o tratamento seguiu as diretrizes da orientação psicodinâmica, sem a utilização de um manual ou protocolo. A partir de diferentes medidas psicométricas, do relato da terapeuta, da mãe e da professora da paciente, 
esse caso foi considerado bem-sucedido. Os resultados foram apresentados de forma detalhada por Gastaud, Carvalho, Fiorini e Ramires (2017).

\section{Instrumento}

Para a análise de sessões, foi utilizado o Child Psychotherapy Q-Set (CPQ; Schneider \& Jones, 2009, 2012), instrumento que avalia o processo psicoterápico de crianças de três a 13 anos de idade. O CPQ é composto por 100 itens que contêm descrições de características relevantes do que pode acontecer em uma sessão de psicoterapia. Os itens são organizados em cartões individuais e representam: (1) os estados emocionais, atitudes, comportamentos ou experiências da criança; (2) as ações e atitudes do terapeuta; e (3) o clima ou atmosfera da sessão ou natureza da interação entre a díade. No manual do CPQ, há definições, descrições e exemplos detalhados sobre os itens, voltados a minimizar diferentes interpretações sobre eles.

O processo de classificação do CPQ é individual e ocorre em duas etapas. Em um primeiro momento, o avaliador assiste à filmagem de uma sessão a ser codificada e chega a alguma formulação do material (i.e., anotações sobre aspectos relevantes). Em um segundo passo, esse avaliador ordena os 100 itens do CPQ em nove categorias, colocando em um extremo os cartões cujas afirmações são mais características ou expressivas em relação à sessão analisada e, no outro, os cartões menos característicos. No centro, são posicionados os itens neutros ou irrelevantes em relação à sessão. Essa ordenação ocorre de forma forçada, ou seja, há uma quantidade fixa de itens que devem ser colocados em cada categoria. Ao final da avaliação, a disposição dos itens forma uma curva normal, com uma quantidade menor de itens nas extremidades e uma concentração maior de itens no centro.

O CPQ foi traduzido e adaptado para o português brasileiro por Ramires e Schneider (2016). Em estudos nacionais e internacionais, o CPQ apresentou bons índices de confiabilidade (Goodman \& Athey-Lloyd, 2011; Goodman et al., 2016; Ramires, Carvalho, Schmidt, Fiorini, \& Goodman, 2015; Schneider, Midgley, \& Duncan, 2010).

\section{Coleta e análise de dados}

Todas as sessões da psicoterapia foram gravadas em vídeo, com o consentimento da paciente, de sua mãe e da terapeuta. Cada sessão foi avaliada independentemente por dois juízes treinados para o seu uso, sendo esses psicólogos ou estudantes de Psicologia. A concordância entre avaliadores, calculada por meio do alfa de Cronbach, variou entre 0,69 a 0,90 $(\mathrm{m}=0,80 ; \mathrm{DP}=0,05)$, considerando todas as 40 sessões da psicoterapia. Após as avaliações individuais, foi calculada a média entre os escores da dupla, formando um perfil de cada sessão. Esses perfis foram utilizados nas análises subsequentes.

A fim de investigar a progressão do processo terapêutico ao longo do tempo, as médias das sessões foram agrupadas em quatro etapas: o período inicial, correspondente ao intervalo entre a primeira e sexta sessão, definiu o estágio em que a terapeuta não estava grávida, ou estava e não sabia. A segunda etapa, ocorrida entre a sétima e 16. ${ }^{a}$ sessão, configurou o período em que a terapeuta sabia que estava grávida, porém esse tema ainda não havia sido mencionado nas sessões. O terceiro

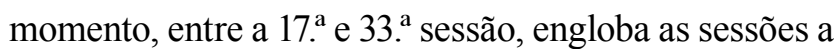
partir da revelação da gravidez à paciente até a licença maternidade. A quarta e última etapa, da 34. a 40 . $^{\text {a }}$ sessão, corresponde ao retorno da licença maternidade até o final do tratamento. Essa divisão foi adotada a partir de apontamentos da literatura, em que a gravidez da terapeuta é vista como um fator que pode impactar no processo terapêutico (Schmidt, Fiorini, \& Ramires, 2015), e entendendo que a gestação da terapeuta de Alice gerou diferentes mobilizações na paciente ao longo do tempo.

Para investigar a similaridade do processo terapêutico em cada período aos protótipos de psicoterapia, foi utilizada a correlação de Pearson. Por fim, o teste t para amostras pareadas foi utilizado para investigar a relação entre os graus de similaridade aos diferentes 
protótipos. Todos as análises foram realizadas por meio do Statistical Package for Social Sciences, versão 24.0.

\section{Protótipos de psicoterapia de crianças}

Para a realização dos cálculos de similaridade do presente estudo, foram utilizados protótipos de PP para crianças com transtornos internalizantes, PP para crianças com transtornos externalizantes, de Terapia CognitivoComportmental (TCC). Ambos os protótipos da PP e o da TCC foram os desenvolvidos por Fiorini e Ramires (2019), a partir da avaliação de experts brasileiros.

\section{Resultados}

A análise descritiva do processo da psicoterapia de Alice, avaliada por meio do CPQ, foi retratada no estudo de Schmidt, Gastaud e Ramires (2018). No presente estudo, foram focalizados os graus de similaridade desse processo psicoterápico aos protótipos brasileiros de $\mathrm{PP}$ e TCC para crianças.

Os escores médios de cada etapa foram submetidos a uma correlação de Pearson com os protótipos de psicoterapia. A progressão dos índices ao longo do tempo é apresentada na Figura 1. A partir dos cálculos realizados, foi verificado que a terapia da paciente, intencionalmente psicodinâmica, apresentou similaridade estatisticamente significativa ao protótipo brasileiro da PP para crianças com transtornos internalizantes em todas as suas etapas $(r=0,62$ a 0,66 ; média $=0,64 ; p$ $<0,01)$. Por outro lado, o processo como um todo não apresentou similaridade ao protótipo brasileiro da PP para crianças com transtornos externalizantes. Apenas no período prévio à licença maternidade o processo apresentou similaridade significativa a este protótipo, porém em menor intensidade $(r=0,21 ; \mathrm{p}<0,05)$.

Ademais, as sessões de psicoterapia de Alice também mostraram similaridade ao protótipo brasileiro da TCC em todas as suas etapas $(r=0,48$ a 0,51 ; média $=$ $0,49 ; \mathrm{p}<0,01)$. Complementarmente, foi verificado que a similaridade ao protótipo da PP para crianças com transtornos internalizantes foi significativamente maior do que ao protótipo da TCC $(\mathrm{t}=-10,079 ; \mathrm{p}<0,002)$.

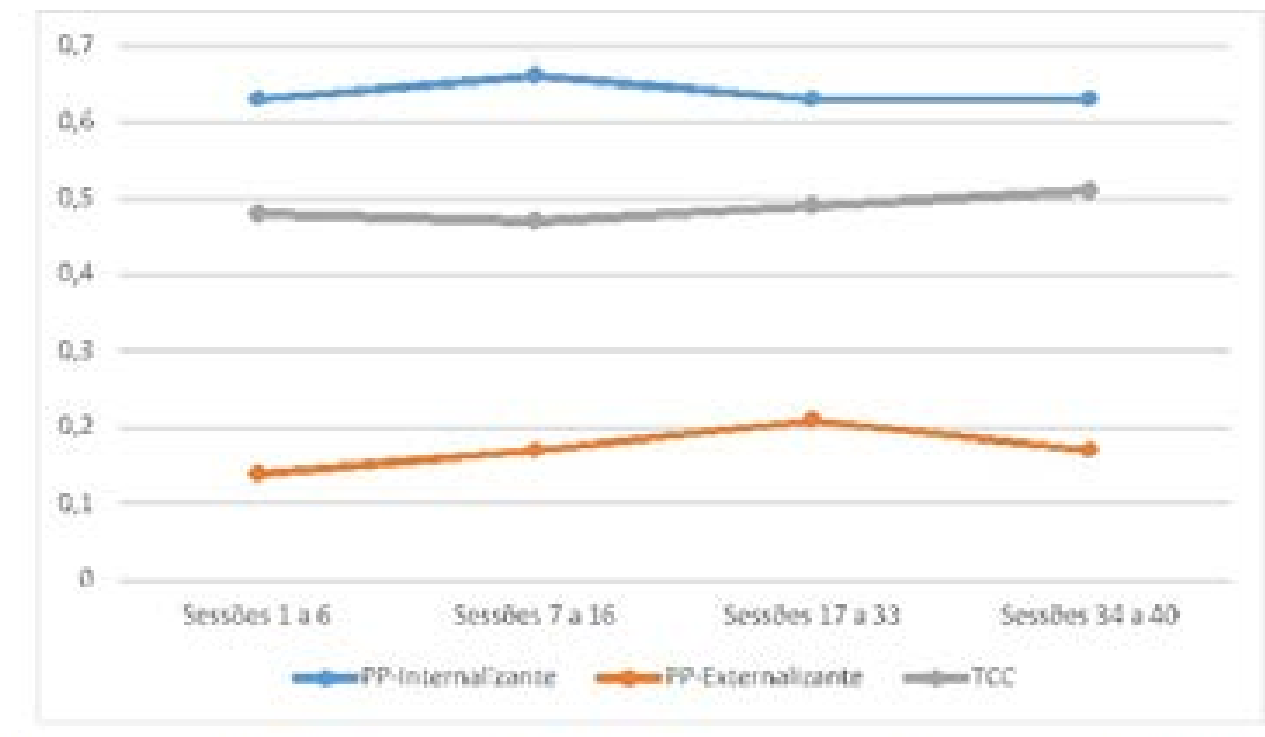

Figura 1. Sümilaridade aos protótipos em relaçăo ao tempo. pp.Internalizante: protótipo be asileiro de Psicoterapia Psicodieh̀mica para crianças coen transtornes iatemalizantes, PP-Externalizante protólpo brasileiro de Psicoterapia Psicodnimaica para crianças corn transtornos externalizantes; TCC protótipo brasal eiro de Terapia Cognitivo Comportamental; 


\section{Discussão}

Este estudo acerca da psicoterapia psicodinâmica de uma criança com sintomas internalizantes foi o primeiro a utilizar os protótipos brasileiros de psicoterapia de crianças para avaliar um tratamento real. A partir da investigação do processo psicoterápico psicodinâmico de Alice, cuja terapeuta engravidou ao longo do tratamento, foram identificados diferentes padrões de similaridade aos protótipos.

Como esperado, todas as fases da psicoterapia de Alice apresentaram similaridade significativa ao protótipo da PP para crianças com transtornos internalizantes, confirmando a hipótese 1. Esse achado indica que o processo psicoterapêutico se desenvolveu de maneira coerente com o modelo teórico que o orientava.

Em consonância ao protótipo da PP para crianças com transtornos internalizantes, a terapeuta constantemente interpretou elementos transferenciais nas sessões, característica presente em todo o processo terapêutico e acentuada no período da gravidez. A medida em que Alice presenciava a progressão gradual da gestação de sua terapeuta, vieram à tona conflitos em relação às suas experiências de separação (com o pai, colegas e, futuramente, com a terapeuta), às suas fantasias quanto à sexualidade e angústias obsessivas. Assim como apontado na literatura (Schmidt et al., 2015), a gravidez da terapeuta pareceu ter sido um catalisador no processo terapêutico, potencializando o trabalho de diferentes temáticas.

Além disso, a similaridade a esse protótipo também pode ser entendida pelas características de Alice, que se apresentou aberta e disposta a examinar e discutir os seus pensamentos e fantasias, permitindo a investigação de seus materiais inconscientes. As particularidades da paciente também podem explicar a alta similaridade logo na primeira etapa do tratamento, no qual geralmente são abordados assuntos como o motivo da consulta e regras do setting para, depois, no período intermediário, haver um aumento no trabalho interpretativo do brincar e das defesas, por exemplo (Etchegoyen, 2005; Luz, 2015).
Em relação à similaridade ao protótipo da PP para crianças com transtornos externalizantes, o processo psicoterapêutico de Alice não apresentou correlação significativa no primeiro, segundo e quarto períodos. No terceiro período, anterior à licença maternidade, o processo apresentou correlação significativa com esse protótipo, porém com baixa intensidade. Da mesma forma que o trabalho interpretativo da terapeuta explicou parcialmente a alta similaridade deste tratamento ao protótipo da PP para crianças com transtornos internalizantes, esse mesmo padrão interventivo explicou parcialmente a baixa similaridade ao protótipo da PP para crianças com transtornos externalizantes.

Ao contrário do que era esperado para o tratamento de Alice, o foco de trabalho das psicoterapias psicodinâmicas para crianças com transtornos externalizantes tende a ser menos interpretativo e mais suportivo e expressivo (Fiorini \& Ramires, 2019; Kernberg \& Chazan, 1991).

A baixa similaridade ao protótipo da PP para crianças com transtornos externalizantes também pode ser explicada pelas atitudes de Alice. A paciente buscava agradar a terapeuta em diferentes sessões e não se mostrava resistente às intervenções realizadas. Ademais, devido à sua sintomatologia marcada pela internalização, as características do processo terapêutico foram de encontro ao que se espera prototipicamente do processo de uma criança com transtornos externalizantes. Devido ao fato de os protótipos incluírem os 100 itens do CPQ e descreverem o processo como um todo, as expectativas prototípicas também incluem itens referentes à criança. Assim, esse achado reforça a concepção de que o processo psicoterápico prototípico da PP é distinto quanto ao tratamento de crianças com transtornos internalizantes e externalizantes. A hipótese 3 foi parcialmente confirmada.

Contrário às nossas expectativas, o processo terapêutico correlacionou-se significativamente ao protótipo da TCC em todos os seus estágios, com uma tendência crescente nas etapas anterior e posterior à licença maternidade. Apesar de o tratamento não apresentar 
características cognitivo-comportamentais típicas, como a estruturação do setting por parte da terapeuta e a utilização de dever de casa (Alford \& Beck, 1997), por exemplo, outras particularidades podem explicar a similaridade a esse protótipo. O foco de discussão sobre questões atuais da criança - como o luto ou ajustamento à escola nova -, as crenças de Alice acerca da sexualidade e a gravidez da terapeuta podem explicar a proximidade do protótipo da TCC. Ressalta-se que a correlação ao protótipo da TCC foi significativamente inferior ao do protótipo da PP para crianças com transtornos internalizantes, podendo indicar que a similaridade encontrada reflete majoritariamente a presença de fatores comuns entre essas abordagens. A hipótese 2 foi refutada.

Tendo em vista as associações significativas deste processo terapêutico a modelos de processos de diferentes perspectivas teóricas, destaca-se um possível caráter integrativo das psicoterapias de crianças. Como indicado por Goodman et al. (2016), os fatores da relação terapêutica são mais evidentes na psicoterapia de crianças e estabelecem um papel mais central no processo. Estudos brasileiros também vêm evidenciando essa tendência à integração: o terapeuta tende a adaptar o processo terapêutico a partir das necessidades da criança, em vez de adequar a criança aos seus pressupostos teóricos (Gastaud et al., 2015; Ramires et al., 2017).

Por outro lado, não há estudos que indiquem que o mesmo padrão ocorra na psicoterapia cognitivo-comportamental de crianças. No estudo de Serralta e Ablon (2016), por exemplo, em que foram avaliados um caso de PP e outro de TCC com pacientes adultas, o tratamento psicodinâmico mostrou-se mais integrativo, enquanto a terapia cognitivo-comportamental apresentou similaridade apenas ao protótipo da respectiva abordagem.

\section{Considerações finais}

No presente estudo, foi avaliado o processo psicoterápico de uma menina com sintomas internalizantes em relação a sua similaridade a protótipos de psico- terapia. Assim como os protótipos internacionais, já empregados em outros estudos (i.e., Gastaud et al., 2015; Goodman, Anderson, \& Diener, 2014; Ramires et al., 2017), os protótipos desenvolvidos em contexto brasileiro demonstraram-se úteis na investigação do processo terapêutico, identificando características-chave do processo avaliado.

Considerando a identificação da similaridade a diferentes modelos psicoterápicos em todas as etapas do tratamento foi reforçado que, na prática clínica, a distinção entre as abordagens terapêuticas não é nítida, e a relação entre fatores comuns e específicos não é dicotômica, como apontado por Weinberger (2014). Ademais, os padrões de similaridade encontrados aos protótipos de PP para crianças com transtornos internalizantes e externalizantes indicam que há diferenças significativas no processo terapêutico dessa mesma abordagem para crianças com as duas dimensões sintomatológicas referidas, reiterando os achados de Fiorini e Ramires (2019).

Os dados encontrados reforçaram que as psicoterapias de crianças, ao menos as psicodinâmicas, tendem a ser, na prática, mais integrativas. Investigações referentes à similaridade de terapias cognitivo-comportamentais de crianças a protótipos poderão contribuir para um maior entendimento desse padrão.

Por fim, ressalta-se que não foram realizadas análises de associação entre a similaridade aos protótipos e os resultados obtidos na psicoterapia neste estudo. Pesquisas futuras que avaliem essa relação poderão indicar quais práticas ou características do processo estão associadas a melhores resultados.

\section{Referências}

Ablon, J. S., \& Jones, E. E. (1998). How expert clinicians' prototypes of an ideal treatment correlate with outcome in psychodynamic and cognitive-behavioral therapy. Psychotherapy Research, 8(1), 71-83. https:// doi.org/10.1080/10503309812331332207

Ablon, J. S., \& Jones, E. E. (2002). Validity of controlled clinical trials of psychotherapy: findings from the 
NIMH treatment of depression collaborative research program. American Journal of Psychiatry, 159(5), 775783. https://doi.org/10.1176/appi.ajp.159.5.775

Achenbach, T. M. (1991). Manual for the Child Behavior Checklist/4-18 and 1991 profile. Burlington: University of Vermont, Department of Psychiatry.

Alford, B. A., \& Beck, A. T. (1997). An analysis of integrative ideology. B. A. Alford \& A. T. Beck (Eds.), The integrative power of cognitive therapy. New York: The Guilford Press.

Chorpita, B. F., Daleiden, E. L., Ebesutani, C., Young, J., Becker, K. D., Nakamura, B. J., ... Starace, N. (2011). Evidence-based treatments for children and adolescents: an updated review of indicators of efficacy and effectiveness. Clinical Psychology: Science and Practice, 18(2), 154-172. https://doi.org/10.1111/j.1468-2850.2011.01247.x

Edwards, D. J. A. (2007). Collaborative Versus Adversarial Stances in Scientific Discourse: Implications for the Role of Systematic Case Studies in the Development of Evidence-Based Practice in Psychotherapy. Pragmatic Case Studies in Psychotherapy, 3(1). https://doi. org/10.14713/pcsp.v3i1.892

Etchegoyen, R. H. (2005). The fundamentals of psychoanalytic technique. London: Karnac Books.

Fiorini, G. P., \& Ramires, V. R. R. (2019). Development of Brazilian prototypes for child psychodynamic psychotherapy and cognitive-behavioral therapy. Trends in Psychiatry and Psychotherapy, 41(2), 149-158. https:// doi.org/10.1590/2237-6089-2018-0053.

Fonagy, P., Bateman, A. W., \& Luyten, P. (2012). Introduction and Overview. In A. W. Bateman \& P. Fonagy (Eds.), Handbook of Mentalizing in Mental Health Practice (pp. 3-42). Washington: American Psychiatric Publishing.

Gastaud, M. B., Carvalho, C., Fiorini, G. P., \& Ramires, V. R. R. (2017). Psychodynamic Psychotherapy of a Child with Internalizing Symptoms: A Study of Outcomes. British Journal of Psychotherapy, 33(4), 514-536. https:// doi.org/10.1590/2237-6089-2014-0059

Gastaud, M. B., Carvalho, C., Goodman, G., \& Ramires, V. R. R. (2015). Assessing levels of similarity to a "psychodynamic prototype" in psychodynamic psychotherapy with children: a case study approach (preliminary findings). Trends in Psychiatry and Psychotherapy, 37(3), 161-165. https://doi.org/10.1590/2237

Goodman, G., Anderson, K., \& Diener, M. J. (2014). Processes of therapeutic change in psychodynamic therapy of two inpatients with borderline personality disorder. Journal of Psychotherapy Integration, 24(1), 30-45. https://doi.org/10.1037/a0035970

Goodman, G., \& Athey-Lloyd, L. (2011). Interaction structures between a child and two therapists in the psychodynamic treatment of a child with Asperger's disorder. Journal of Child Psychotherapy, 37(3), 311-326. https://doi.org/10.1080/0075417X.2011.614749

Goodman, G., Midgley, N., \& Schneider, C. (2016). Expert clinicians' prototypes of an ideal child treatment in psychodynamic and cognitive-behavioral therapy: Is mentalization seen as a common process factor? Psychotherapy Research, 26(5), 590-601. https://doi. org/10.1080/10503307.2015.1049672

Goodman, G., Reed, P., \& Athey-Lloyd, L. (2015). Mentalization and play therapy processes between two therapists and a child with Asperger's disorder. International Journal of Play Therapy, 24(1), 13-29. https://doi.org/10.1037/a0038660

Jones, E. E. (1985). Psychotherapy Process Q-Set Coding Manual. Berkeley. Retrieved from http://www. homepages.ucl.ac.uk/ ucjtaca/pqsmanual.pdf

Jones, E. E., Cumming, J. D., \& Horowitz, M. J. (1988). Another look at the nonspecific hypothesis of therapeutic effectiveness. Journal of Consulting and Clinical Psychology, 56(1), 48-55. https://doi.org/10.1037/ 0022-006X.56.1.48

Kazdin, A. E. (2009). Understanding how and why psychotherapy leads to change. Psychotherapy Research: Journal of the Society for Psychotherapy Research, 19(45), 418-28. https://doi.org/10.1080/10503300802448899

Kernberg, P. B., \& Chazan, S. (1991). Children with conduct disorders: a psychotherapy manual. New York: Basic Books.

Kovacs, M., \& Devlin, B. (1998). Internalizing Disorders in Childhood. Journal of Child Psychology and Psychiatry, 39(1), 47-63. https://doi.org/10.1111/14697610.00303

Leichsenring, F., Ablon, S., Barber, J. P., Beutel, M., Gibbons, M. B. C. B., Crits-Christoph, P., \& Salzer, 
S. (2016). Developing a prototype for short-term psychodynamic (supportive-expressive) therapy: An empirical study with the psychotherapy process Q-set. Psychotherapy Research, 26(4), 500-510. https://doi.or $\mathrm{g} / 10.1080 / 10503307.2015 .1051160$

Leonardi, J. L., \& Meyer, S. B. (2015). Prática baseada em evidências em psicologia e a história da busca pelas provas empíricas da eficácia das psicoterapias. Psicologia: Ciência E Profissão, 35(4), 1139-1156. https://doi.org/10.1590/1982-3703001552014

Luz, A. B. (2015). Fases da psicoterapia. In C. L. Eizirik, R. W. Aguiar, \& S. S. Schestatsky (Eds.), Psicoterapia de orientação analítica (pp. 249-266). Porto Alegre: Artmed.

Midgley, N., O’Keeffe, S., French, L., \& Kennedy, E. (2017). Psychodynamic psychotherapy for children and adolescents: an updated narrative review of the evidence base. Journal of Child Psychotherapy, 37(3), 1-23. https:// doi.org/10.1080/0075417X.2017.1323945

Oland, A. A., \& Shaw, D. S. (2005). Pure versus co-occurring externalizing and internalizing symptoms in children: The potential role of socio-developmental milestones. Clinical Child and Family Psychology Review, 8(4), 247-270. https://doi.org/10.1007/s10567$005-8808-\mathrm{z}$

Organização Mundial da Saúde. (n.d.). Child and adolescent mental health. Retrieved May 1, 2017, from http:// www.who.int/mental_health/maternal-child/child_adolescent/en/ https://doi.org/10.5772/67556

Palmer, R., Nascimento, L. N., \& Fonagy, P. (2013). The state of the evidence base for psychodynamic psychotherapy for children and adolescents. Child and Adolescent Psychiatric Clinics of North America, 22(2), 149-214. https://doi.org/10.1016/j.chc.2012.12.001

Polanczyk, G. V, Salum, G. A., Sugaya, L. S., Caye, A., \& Rohde, L. A. (2015). Annual Research Review: A meta-analysis of the worldwide prevalence of mental disorders in children and adolescents. Journal of Child Psychology and Psychiatry, 56(3), 345-365. https://doi. org/10.1111/jcpp.12381

Ramires, V. R. R., Carvalho, C., Schmidt, F. M. D., Fiorini, G. P., \& Goodman, G. (2015). Interaction structures in the psychodynamic therapy of a boy diagno- sed with Asperger's Disorder: A single-case study. Research in Psychotherapy: Psychopathology, Process and Outcome, 18(2), 129-140. https://doi.org/10.4081/ ripppo.2015.195

Ramires, V. R. R., Godinho, L. B. R., \& Goodman, G. (2017). The therapeutic process of a child diagnosed with disruptive mood dysregulation disorder. Psychoanalytic Psychology, 34(4), 488-498. https://doi.org/10.1037/ pap0000134

Ramires, V. R. R., \& Schneider, C. (2016). Psicoterapia de Crianças: Desenvolvimento da Versão em Português do Child Psychotherapy Q-Set. Psicologia: Teoria E Pesquisa, 32(3), 1-10. https://doi.org/10.1590/ 0102-3772e323218

Recktenvald, K., Mallmann, M. Y., Schmidt, F. M. D., Fiorini, G. P., \& Cappellari, C. P. C. (2016). Caracterização da clientela de bebês em uma clínica-escola de psicoterapia psicanalítica. Revista Brasileira de Psicoterapia, 18(3), 15-30. https://doi.org/10.5935/19806906/psicologia.v19n3p70-83

Sampieri, R. H., Collado, C. F., \& Lucio, M. del P. B. (2014). Metodología de la investigación. City of Mexico: McGraw Hill.

Schmidt, F. M. D., Fiorini, G. P., \& Ramires, V. R. R. (2015). Psychoanalytic psychotherapy and the pregnant therapist: A literature review. Research in Psychotherapy: Psychopathology, Process and Outcome, 18(2), 50-61. https://doi.org/10.4081/ripppo.2015.185

Schmidt, F. M. D., Gastaud, M. B., \& Ramires, V. R. R. (2018). Child Psychodynamic Psychotherapy and Therapist Pregnancy: Systematic Case Study. Paidéia (Ribeirão Preto), 28, 36. https://doi.org/10.1590/ 1982-4327e2836.

Schneider, C., \& Jones, E. E. (2009). Child Psychotherapy $Q$-Set coding manual. Berkeley: University of California. Retrieved from http://www.homepages.ucl.ac.uk/ ucjtaca/ cpqmanual.pdf

Schneider, C., \& Jones, E. E. (2012). Appendix IB. Child Psychotherapy Q-Set. Coding Manual. In R. A. Levy, J. S. Ablon, \& H. Kächele (Eds.), Psychodynamic Psychotherapy Research: Evidence-Based Practice and Practice-Based Evidence (pp. 611-626). New York: Humana Press. https://doi.org/10.1007/978-1-60761-792-1 
Schneider, C., Midgley, N., \& Duncan, A. (2010). A "motion portrait" of a psychodynamic treatment of an 11-year-old girl: exploring interrelations of psychotherapy process and outcome using the child psychotherapy Q-set. Journal of Infant, Child, and Adolescent Psychotherapy, 9(2-3), 94-107. https://doi.org/10.1080 /15289168.2010.510979

Serralta, F. B., \& Ablon, J. S. (2016). Development of Brazilian prototypes for short-term psychotherapies. Trends in Psychiatry and Psychotherapy, 38(2), 71-79. https://doi.org/10.1590/2237-6089-2015-0039

Serralta, F. B., Pole, N., Nunes, M. L. T., Eizirik, C. L., \& Olsen, C. (2010). The process of change in brief psychotherapy: Effects of psychodynamic and cognitive-behavioral prototypes. Psychotherapy Research, 20(5), 564-575. https://doi.org/10.1080/10503307.2010.493537

Shedler, J. (2010). The efficacy of psychodynamic psychotherapy. American Psychologist, 65(2), 98-109. https://doi.org/10.1037/a0018378

Wampold, B. E. (2015). How important are the common factors in psychotherapy? An update. World Psychiatry, 14(3), 270-277. https://doi.org/10.1002/wps.20238

Weinberger, J. (2014). Common factors are not so common and specific factors are not so specified: toward an inclusive integration of psychotherapy research. Psychotherapy, 51(4), 514-518. https://doi.org/10.1037/ a0037092

Wilkinson, P. (2009). Conceptualization about internalizing problems in children and adolescents. Ciência \& Saúde Coletiva, 14(2), 373-381. https://doi.org/10.1590/ S1413-81232009000200007

\section{Endereço para correspondência: Guilherme}

Pacheco Fiorini (Department of Clinical, Educational and Health Psychology - University College London, 1-19 Torrington Place WC1E 7HB - Londres, Reino Unido. E-mail: guilherme.fiorini@outlook.com).

\section{Guilherme Pacheco Fiorini}

Titulação Acadêmica: Mestre.

Afiliação Institucional: Universidade do Vale do

Rio dos Sinos (UNISINOS), University College

London (UCL) e Anna Freud Centre for Children and Families (AFNCCF)

E-mail: Guilherme.fiorini@outlook.com

\section{Marina Bento Gastaud}

Titulação Acadêmica: Doutora.

Afiliação Institucional: Contemporâneo - Instituto de Psicanálise e Transdisciplinaridade

E-mail: marinagastaud@hotmail.com

\section{Vera Regina Röhnelt Ramires.}

Titulação Acadêmica: Doutora.

Afiliação Institucional: Universidade do Vale do Rio dos Sinos (UNISINOS).

E-mail:vramires@unisinos.br

Recebido em: 2/5/2018.

Aceito em: 14/5/2019.

Publicado em: xx/xx/2019. 